\title{
Capillary regression, a key pathogenic feature of COVID-19 infection?
}

\author{
George E. Davis ${ }^{1}$ and Courtney T. Griffin ${ }^{2,3}$
}

1Department of Molecular Pharmacology and Physiology, Morsani College of Medicine, University of South Florida School of Medicine, Tampa, FL 33612

${ }^{2}$ Cardiovascular Biology Research Program, Oklahoma Medical Research Foundation, and ${ }^{3}$ Department of Cell Biology, University of Oklahoma Health Sciences Center, Oklahoma City, OK 73104

Correspondence to:

George E. Davis, M.D., Ph.D.

Professor

Department of Molecular Pharmacology and Physiology

Morsani College of Medicine

University of South Florida

12901 Bruce B. Downs Blvd., MDC8

Tampa, FL 33612

Phone- (813) 974-5985

E-mail-gedavis@usf.edu 


\section{ABSTRACT}

In this brief communication, we propose the concept that capillary regression may represent a primary pathogenic process underlying COVID-19 infection, particularly in the serious and life-threatening manifestations of the disease. We suggest that the marked elevations of pro-inflammatory mediators that are observed in these seriously ill patients may directly induce capillary regression and endothelial cell $(E C)$ loss. Recent autopsy studies are demonstrating EC loss leading to widespread microthrombi and associated tissue damage. Recent work has indicated that interleukin-1 beta (IL-1 $\beta$ ), tumor necrosis factor-alpha (TNF $\alpha$ ), and thrombin, individually and in combination, can potently cause capillary tube regression in experimental models in vitro and in vivo. Other pro-inflammatory mediators including interferon gamma (IFNY), interleukin-4 (IL4), and interleukin-13 (IL-13) were also shown to be pro-regressive and could be relevant mediators in COVID-19 patients. Interestingly, combinations of pharmacologic agents were identified that reduced capillary regression and protected capillary tube networks against these pro-inflammatory mediators. Such an approach might be an important therapeutic option going forward to treat key disease states where capillary regression plays a major underlying pathogenic role. Finally, if capillary regression is occurring in response to these pro-inflammatory mediators during COVID-19 infection, we suggest that combinations of blocking agents directed to these key pro-regressive mediators might be necessary to appropriately treat patients. 


\section{COMMUNICATION}

In severe cases, coronavirus disease 2019 (COVID-19) is associated with acute respiratory distress syndrome (ARDS), which classically results from increased permeability of pulmonary capillaries [1-3]. The vascular leakage characteristic of ARDS diminishes the capacity for oxygen to diffuse from the alveolar space into adjacent capillaries, leading to respiratory failure and systemic hypoxia. Other mechanisms that can contribute to capillary dysfunction and hypoxia include development of microthrombi and endothelial cell (EC) death. COVID-19 autopsy cases are revealing evidence for all of these mechanisms of pulmonary capillary damage, including microthrombi, inflammatory cell infiltration into small vessels, EC death, edema, and focal hemorrhages [1, 2, 4, 5]. In this brief perspective, we address potential mechanisms by which COVID-19 might lead to EC death and capillary regression in lungs and other organs, resulting in an ARDS-like condition, systemic hypoxia and multi-organ failure.

Patients with particularly severe COVID-19 symptoms frequently show marked elevation of pro-inflammatory factors such as thrombin, IFNy, and the cytokines TNFa, IL-6, and IL-1 $[3,6-8]$. These factors are interesting in the context of COVID-19-related vascular damage because of their known capacity to cause vascular permeability and/or thrombosis. They are also interesting to our two laboratories because of our recent work identifying key regulators of capillary regression [9]. Capillary regression (i.e. EC loss and death) is an understudied process that appears to play a pathogenic role in numerous disease states, including hypertension, ischemia and infarction, diabetes, heart failure, malignant cancers, neurodegeneration, and sepsis. Our broad screen of many growth factors, cytokines and other molecules revealed that the most potent proregressive molecules we could identify were IL-1 $\beta$, IL-1 $\alpha$, TNF $\alpha$, and thrombin [9]. When added individually or in combination, they induced profound capillary collapse and regression and selective loss of EC-lined tubes but did not cause loss of associated pericytes [9]. Thrombin also enhanced the pro-regressive activities of both IL-1 isoforms and TNFa. This broad screen also revealed that IFNy and IL-4 have strong pro-regressive activity, and both have been identified as upregulated factors in the 
COVID-19 "cytokine storm". Interestingly, with regard to current COVID-19 clinical trials, the addition of IL-6 did not cause regression of capillary tubes in these experiments [9]. Importantly, this work also identified strong pro-regressive activities derived from activated macrophages treated with the toll-like receptor agonist lipopolysaccharide (LPS), a component derived from Gram-negative bacteria. Conditioned medium from LPS-treated macrophages dramatically induced capillary regression of EC tube networks, and antibody neutralization experiments demonstrated that the key proregressive factors made by the macrophages were IL-1 $\beta$ and TNFa [9]. Blocking antibodies to these two factors interfered with the macrophage pro-regressive activity, while neutralizing antibodies directed to IL- 6 or IL-1 $\alpha$ had no effect. This finding was further validated when blocking antibodies directed to both IL-1 $\beta$ and TNFa led to reduced hyaloid vascular regression (a physiological vascular regression process) in the neonatal mouse eye [9]. Hyaloid vessel regression relies on local macrophages, which we found induce IL-1 $\beta$ during the regression process. Overall, this work demonstrated that capillary regression is potently controlled by macrophage-derived pro-inflammatory cytokines (i.e. IL-1 $\beta$ and TNF $\alpha$ ) and that the pro-regressive activity of these cytokines is further enhanced by thrombin. Interestingly, there has been a recent call to use antiTNF therapy in COVID-19 patients [10] and furthermore, the administration of IL-1 receptor antagonist (IL-1RA) to a pediatric patient with COVID-19 appeared to have a clinical benefit [11].

Because of the correlation between factors upregulated in severely ill COVID-19 patients and those we identified that cause capillary regression, a central question is whether the SARS-CoV-2 virus can induce capillary regression in infected tissues such as the lung and heart, which are essential for systemic oxygenation and tissue health (Figure 1). The alveolar wall, a major site of infection for SARS-CoV-2 and other upper respiratory viruses, consists of alveolar epithelial cells, adjacent macrophages, and capillaries consisting of ECs and associated pericytes. Macrophages are a major source of inflammatory cytokines, including the ones that we have found to trigger capillary regression. Interestingly, SARS-coronaviruses and many other highly pathogenic viruses can activate the NLRP3 inflammasome, which leads to production and release of IL-1 $\beta$ and IL-18 from various cell types, including macrophages $[3,6]$. Therefore, 
direct infection of macrophages with SARS-CoV-2, or interactions between macrophages and other infected cell types (e.g. in the alveolar wall), might induce inflammasome activation and local elevation of active IL-1 $\beta$ as well as upregulate the production and release of other key mediators such as TNF $\alpha$ and thrombin that could drive capillary regression $[6,9]$.

An autopsy study of COVID-19 patients revealed the presence of viral particles within ECs, and these patients showed evidence of EC apoptosis and inflammation associated with degenerating ECs [2, 5]. In addition, microthrombi were observed within the lumens of many small vessels. Additional autopsy studies also presented evidence for EC dysfunction as a central pathogenic feature in many tissues, including the lung, heart, kidney and small intestine $[1,4,5]$. In these tissue sites, microthrombi, focal necrosis, and hemorrhages were observed. Evidence for ARDS within the lung tissues of these patients was also noted, including diffuse alveolar damage, hyaline membranes in alveolar spaces, and thickening of alveolar interstitial spaces. A key question is whether the capillary disruption and EC death observed in these patients resulted directly from viral infection of ECs or from inflammatory mediator-induced capillary collapse and regression. The early autopsy findings are consistent with the possibility that capillary regression is occurring and that it may be a central pathogenic feature of this disease process. A capillary regression signaling signature we recently identified [9] could help with determining if capillary regression is occurring in COVID-19 patients. We speculate that the ultimate severity of the disease might relate to the extent of capillary loss during the infection coupled to the basal state of pulmonary capillary density and function that was present in patients prior to viral infection (Figure 1). Such considerations might explain the findings of associated pre-existing conditions correlating with COVID-19 disease severity, including cardiovascular diseases such as hypertension or heart failure, diabetes, obesity, age, and smoking status, which can be associated with reduced functional capillary networks within the pulmonary vasculature and other tissues [1] (Figure 1).

If capillary regression is indeed a key aspect of COVID-19 pathogenesis, this will impact therapeutic options for patients. In contrast to the transient issue of capillary 
leakage, which is reversible, physical loss of ECs through regression is a distinct and more complex therapeutic problem. Thus, an important new therapeutic strategy to consider for COVID-19 patients is protecting against EC death and capillary regression (Figure 1). Since the combination of IL-1 $\beta, T N F \alpha$, and thrombin causes capillary tube network regression by inducing selective EC apoptosis (without loss of pericytes) [9], combined blockade of these molecules (i.e. through the use of blocking antibodies, IL1RA, Factor Xa inhibitors or PAR1 inhibitors) may be necessary to prevent capillary regression caused by SARS-CoV-2 infection. Since additional inflammatory mediators such as IFNy, IL-4, and IL-13 can also disrupt capillary networks [9], blockade of these molecules (i.e. with blocking antibodies) may also be necessary to prevent the capillary regression process. We hypothesize that in serious acute injury situations (e.g. COVID19 , ischemia, infarction, burn injury, or sepsis), multiple inflammatory mediators released in a "cytokine storm" may contribute to capillary regression, and more effective therapies will require inhibition of these mediators in combination rather than just individually.

An alternative strategy would be to develop therapeutics to protect capillary tube networks by antagonizing pro-regression signaling pathways[9] with pharmacologic agents (Figure 1). This approach is analogous to what has been utilized in therapies for ARDS or sepsis to reduce capillary permeability. Recently, we identified a combination of pharmacologic drugs (i.e. abbreviated FISTSB) that interfered with EC tube regression in response to single or multiple pro-regressive mediators, including IL$1 \beta$, TNF $\alpha$, and thrombin (also IFNy, IL-4, and IL-13) [9]. Pharmacologic agents such as these might be particularly effective in alleviating functional or physical capillary loss in COVID-19 patients when used in combination with neutralizing antibodies or antagonistic recombinant proteins (e.g. IL-1RA) against pro-regressive inflammatory mediators (Figure 1). Finally, the development of such therapeutic strategies should be broadly applicable to other disease states where capillary regression is a major underlying pathogenic mechanism. 


\section{$\underline{\text { References }}$}

1. Menter T, Haslbauer JD, Nienhold R, Savic S, Hopfer H, Deigendesch N, et al. Post-mortem examination of COVID19 patients reveals diffuse alveolar damage with severe capillary congestion and variegated findings of lungs and other organs suggesting vascular dysfunction. Histopathology. 2020. doi: 10.1111/his.14134. PubMed PMID: 32364264.

2. Varga Z, Flammer AJ, Steiger $P$, Haberecker $M$, Andermatt R, Zinkernagel AS, et al. Endothelial cell infection and endotheliitis in COVID-19. Lancet. 2020;395(10234):1417-8. doi: 10.1016/S0140-6736(20)30937-5. PubMed PMID: 32325026; PubMed Central PMCID: PMCPMC7172722.

3. Henderson LA, Canna SW, Schulert GS, Volpi S, Lee PY, Kernan KF, et al. On the Alert for Cytokine Storm: Immunopathology in COVID-19. Arthritis Rheumatol. 2020. doi: 10.1002/art.41285. PubMed PMID: 32293098.

4. Wichmann D, Sperhake JP, Lutgehetmann M, Steurer S, Edler C, Heinemann A, et al. Autopsy Findings and Venous Thromboembolism in Patients With COVID-19: A Prospective Cohort Study. Ann Intern Med. 2020. doi: 10.7326/M20-2003. PubMed PMID: 32374815.

5. Ackermann M, Verleden SE, Kuehnel M, Haverich A, Welte T, Laenger F, et al. Pulmonary Vascular Endothelialitis, Thrombosis, and Angiogenesis in Covid-19. N Engl J Med. 2020. doi: 10.1056/NEJMoa2015432. PubMed PMID: 32437596.

6. Merad M, Martin JC. Pathological inflammation in patients with COVID-19: a key role for monocytes and macrophages. Nat Rev Immunol. 2020. doi: 10.1038/s41577020-0331-4. PubMed PMID: 32376901; PubMed Central PMCID: PMCPMC7201395. 7. Mehta P, McAuley DF, Brown M, Sanchez E, Tattersall RS, Manson JJ, et al. COVID-19: consider cytokine storm syndromes and immunosuppression. Lancet. 2020;395(10229):1033-4. doi: 10.1016/S0140-6736(20)30628-0. PubMed PMID: 32192578.

8. Pedersen SF, Ho YC. SARS-CoV-2: a storm is raging. J Clin Invest. 2020;130(5):2202-5. doi: 10.1172/JCI137647. PubMed PMID: 32217834.

9. Koller GM, Schafer C, Kemp SS, Aguera KN, Lin PK, Forgy JC, et al. Proinflammatory Mediators, IL (Interleukin)-1beta, TNF (Tumor Necrosis Factor) alpha, 
and Thrombin Directly Induce Capillary Tube Regression. Arterioscler Thromb Vasc Biol. 2020;40(2):365-77. doi: 10.1161/ATVBAHA.119.313536. PubMed PMID: 31852224.

10. Feldmann M, Maini RN, Woody JN, Holgate ST, Winter G, Rowland M, et al. Trials of anti-tumour necrosis factor therapy for COVID-19 are urgently needed. Lancet. 2020;395(10234):1407-9. doi: 10.1016/S0140-6736(20)30858-8. PubMed PMID: 32278362; PubMed Central PMCID: PMCPMC7158940.

11. Pain CE, Felsenstein S, Cleary G, Mayell S, Conrad K, Harave S, et al. Novel paediatric presentation of COVID-19 with ARDS and cytokine storm syndrome without respiratory symptoms. Lancet Rheumatol. 2020. doi: 10.1016/S2665-9913(20)30137-5. PubMed PMID: 32427161; PubMed Central PMCID: PMCPMC7228732.

\section{Figure Legends}

Figure 1. Potential role of pro-inflammatory mediator-induced capillary regression in COVID-19 and other related disease states.

\section{Conflicts of Interest}

The authors declare no conflicts of interest regarding this manuscript. 


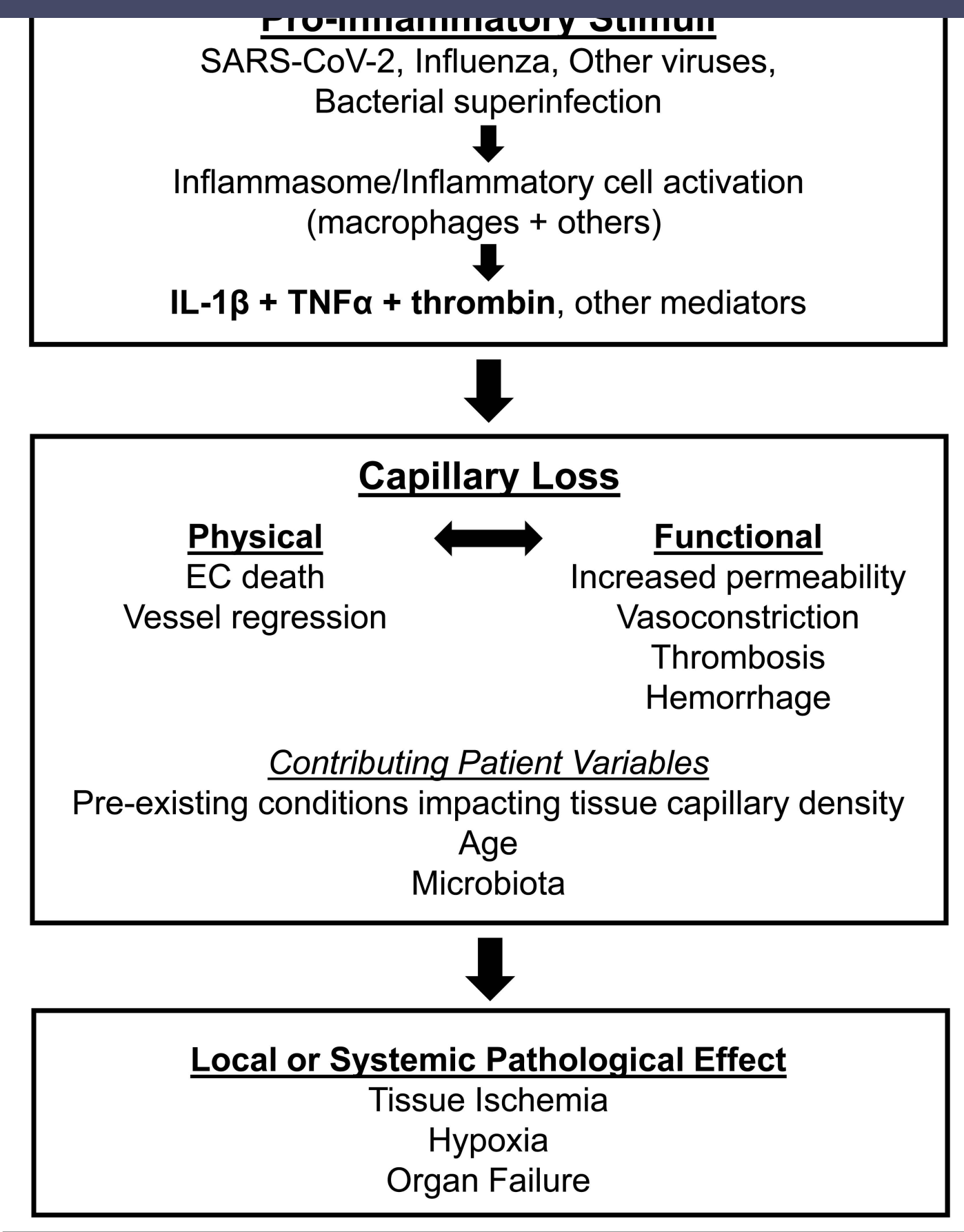

\section{Therapeutic Options and Goals}

Combinations of pharmacological inhibitors, neutralizing antibodies, and recombinant proteins:

\section{Blockade of capillary loss (Capillary Protection)} to reduce vascular permeability, inhibit capillary regression, and maintain/ enhance tissue oxygenation 